\title{
Multiple pelvic accessory spleen: Rare case report with review of literature
}

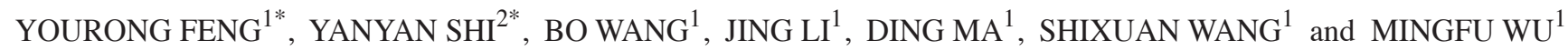 \\ ${ }^{1}$ Department of Gynaecology, Cancer Biology Research Center, Tongji Hospital; ${ }^{2}$ Department of Anesthesiology, \\ Wuhan Pu'ai Hospital, Tongji Medical College, Huazhong University of \\ Science and Technology, Wuhan, Hubei 430030, P.R. China
}

Received October 10, 2017; Accepted January 10, 2018

DOI: $10.3892 /$ etm.2018.5903

\begin{abstract}
Accessory spleen (AS) usually presents as an isolated asymptomatic mass of splenic tissue separated from the body of the actual spleen. Multiple pelvic ASs are more unusual than single pelvic AS. The present study reported on the rare case of multiple pelvic AS. A 39-year-old Chinese woman presented at our hospital with complaints of abdominal pain for one week. A large pelvic mass behind the uterus was identified on ultrasound examination. Multiple AS in the pelvis was primarily considered during the operation and confirmed by histopathological examination after the surgery. The literature in English language was also reviewed by retrieving studies on AS published over the past 30 years, and it was discussed how to diagnose and treat pelvic AS. In conclusion, to the best of our knowledge, the present study provided the first case report of multiple pelvic AS. The gynecologist should be aware of the rare possibility of an AS in patients with abdominal and pelvic complaints and/or a pelvic mass. Pelvic AS is generally determined during radiological investigations or during open or laparoscopic surgeries.
\end{abstract}

\section{Introduction}

Accessory spleens (AS), also known as supernumerary spleen, splenules or splenunculi, are commonly accepted as congenital abnormalities due to the failing of fusion of the splenic primordium, originating from the left side of the dorsal mesogastrium during the initial phases of fetal life (1). Although AS affects $10-30 \%$ of the population (2), its pre-operative diagnosis by

Correspondence to: Professor Mingfu Wu, Department of Gynaecology, Cancer Biology Research Center, Tongji Hospital, 1095 Jiefang Road, Wuhan, Hubei 430030, P.R. China

E-mail: mingfuwutj@163.com

${ }^{*}$ Contributed equally

Abbreviations: AS, accessory spleen; CT, computed tomography

Key words: accessory spleen, pelvis, differential diagnosis gynecologists is challenging due to the rare incidence of AS located in the pelvis and the asymptomatic pattern of its manifestation. The present study reports on the case of a pelvic multiple AS appearing in the para-uterus and the junction between the double sacral ligament and the posterior wall of the cervix but no presence of any adnexal mass. The literature was also reviewed by retrieving and summarizing studies on pelvic AS from the past 30 years. To the best of our knowledge, no previous study has comprehensively described multiple AS occurring in the pelvis as well as its differential diagnoses of gynecological diseases.

\section{Case report}

A 39-year-old woman with sudden abdominal pain for one week presented at the Department of Gynaecology, Tongji Hospital (Wuhan, China) in July 2017. She had a big pelvic mass behind the uterine that had been incidentally detected during a routine ultrasound scan test two months ago, but no adnexal mass was identified. Laboratory tests indicated no abnormality, and tumor bio-markers cancer antigen (CA)125, $\alpha$-fetoprotein and carcinoembryonic antigen (CEA) were within normal limits. Transvaginal color doppler ultrasonography revealed a post-uterine mass with a maximum diameter of $73 \mathrm{~mm}$, which presented a homogeneously hypoechoic pattern and demonstrated no pathological blood flow (Fig. 1A and B). The patient's medical history revealed no surgery of the spleen due to any upper abdominal trauma.

Due to the sudden abdominal pain and the big pelvic mass, a laparotomy was performed under general anesthesia by non-invasive procedures in order to reveal the nature of the pelvic mass and to rule out the suspected diagnosis of pelvic neoplasm or gastrointestinal tumor. Inspection of the pelvis revealed multiple solid and firm tumors with a smooth surface of $2-8 \mathrm{~cm}$ in diameter. Apart from a $2-\mathrm{cm}$ diameter kermesinus and pediculated mass next to the left posterior wall of the uterus, 2 further big masses of $\sim 8 \mathrm{~cm}$ in diameter, located next to the junction between the bilateral utero-sacral ligament and the posterior wall of the cervix, were present (Fig. 1C and D). There was no adhesion to the surrounding structures. The bilateral salpinx, ovary and the uterus were normal on inspection. The mass next to the left posterior wall of the uterus was resected, following which the vascular 
Table I. The differences between multiple pelvic AS and single pelvic AS.

\begin{tabular}{lll}
\hline Characteristic & \multicolumn{1}{c}{ Multiple pelvic AS } & Single pelvic AS \\
\hline Quantity & $\geq 2$ & 1 \\
Frequency of the position & Left broad ligament and Douglas or obturator fossa & Adnexal region \\
Incidence & Extremely rare & Rare \\
$\begin{array}{l}\text { Clinical features during medical } \\
\text { detection or operation }\end{array}$ & Multiple masses & Single mass \\
$\begin{array}{l}\text { Diagnosis } \\
\text { Differential diagnosis }\end{array}$ & Difficult & Easy \\
\hline
\end{tabular}

AS, accessory spleen.
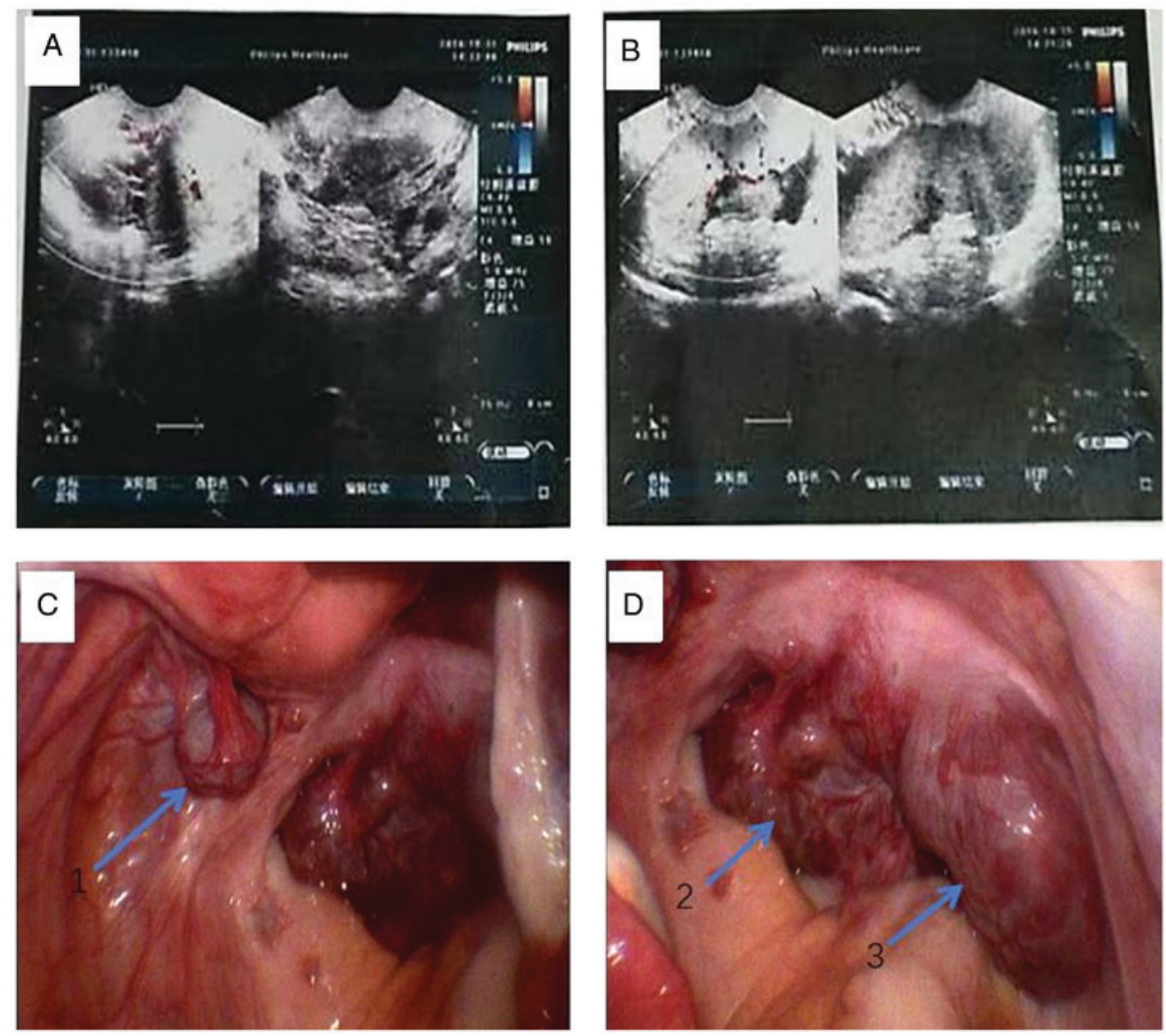

Figure 1. (A) Sagittal gray-scale transvaginal sonogram of the right pelvic area, demonstrating the solid, homogeneous pelvic mass. (B) Color Doppler sonogram revealing the large, relatively straight vessels inside the mass. (C and D) Inspection of the pelvis on laparoscopy revealed multiple solid and firm tumors with a smooth surface, $2-8 \mathrm{~cm}$ in diameter. Apart from (C) a 2-cm diameter kermesinus and pediculated mass next to the posterior wall of the uterus, (D) the other two big masses, $\sim 8 \mathrm{~cm}$ in diameter, were located at the junction between the bilateral utero-sacral ligament and the posterior wall of the cervix.

peduncle was isolated and ligated during an open surgery. Pathological examination of the resected specimens revealed splenic tissue. Post-operative recovery was uneventful.

\section{Discussion}

AS refers to one or more ectopic splenic tissue masses with a similar structure and the same endothelial function to that of the spleen, which is in turn present in its normal position. In the clinic, AS is detected incidentally and has no clinical manifestation or causes slight discomfort in most patients. AS is present in 10-30\% of the general population according to an autopsy-based study (3). In atypical cases, the pre-operative diagnosis of AS is difficult.
To date, the only reported case of a large pelvic AS was that of a juvenile female with a wandering congestive mass $(13 \times 8 \times 7 \mathrm{~cm})$ with a tortuous vascular pedicle that originated along the dorsal aspect of the greater omentum situated in the cul-de-sac (4). The present study reported on another case of a pelvic large AS in a 39-year-old woman presenting with multiple solid masses.

Ectopic splenic tissue may be either congenital (AS or splenunculi) or acquired (splenosis). Splenosis is the auto-transplantation of splenic tissue during splenectomy or following trauma (5). The patient of the present study had no history of trauma or splenectomy. AS is a congenital defect defined as additional ectopic splenic parenchyma (2). The size of the AS ranges from microscopic to $4.5 \mathrm{~cm}$ in diameter, as observed 
in previously reported cases $(2,6,7)$. The mean age of cases is 20-40 years according to one study (6), while the youngest reported case was 17 years $(4,8)$ and the oldest was 67 years $(9)$. The localization varies widely, but the most common locations are the splenic hilum (75\%) or the pancreas tail (20\%). They may infrequently be located in the greater omentum, mesenterium, adnexal region, the pouch of Douglas, the obturator fossa and retroperitoneal area, as well as the pelvic cavity $(2,10)$. Unver Dogan et al (11) investigated 720 autopsy cases, among which AS was present in $6.7 \%$ of cases, 2 of which were pelvic AS. The common presentation is that of a single AS (85\%), although 20 (14\%) and rarely $\geq 3$ (1\%) may also be observed (5).

AS located in the pelvis (named as pelvic AS) is not frequently seen. Multiple pelvic AS is defined as two or more pelvic AS located to the left broad ligament and the pouch of Douglas or the obturator fossa except for the adnexal region. A total of 6 differences prevail between multiple pelvic AS and single pelvic AS (Table I). Pelvic AS may be divided into two conditions: One is the wandering spleen, which is a separate entity, resulting from an elongation or a defect due to the incomplete development of the ligamentous splenic apparatus, which is caused by congenital defects during dorsal mesogastrium development or by acquired defects, such as abdominal wall laxity, which allow the spleen to migrate within the abdomen (6). The other condition is splenosis, which is an acquired abnormality relevant to heterotopic auto-transplantation of splenic tissue following abdominal trauma or splenectomy (7). According to the literature in English language of the past 30 years (from 1987 to 2017) retrieved online from PubMed (https://www.ncbi.nlm.nih. gov/pubmed/), only 16 cases of pelvic AS have been reported, to the best of our knowledge (2,4-10,12-19).

Although most patients with pelvic AS are asymptomatic, symptomatic pelvic AS rarely presents clinically as an abdominal mass causing complications, including torsion, spontaneous rupture, hemorrhage or cyst formation $(6-8,13,16)$. Torsion and ischemia of pelvic AS may lead to acute abdomen $(13,16)$. When the AS is located in the adnexal area, the differential diagnoses of this adnexal solid mass comprise the different causes of adnexal masses, including enlarged lymph nodes, sub-serous fibroid, ovarian tumors, organized hematoma and tubo-ovarian abscess $(5,13,18)$.

As pelvic AS often mimics adnexal masses or malignancy (e.g., ovarian tumors or organized hematoma) with plentiful pulsating blood flow, it may be misdiagnosed during medical imaging. As pelvic AS does not require treatment unless it is symptomatic or torsion and infarction of asymptomatic AS occur, accurate diagnosis is important. Thus, differentiation of this benign splenic abnormality from pathological disorders at the pre-operative stage is often a diagnostic dilemma for gynecologists (5). AS is generally detected during radiological investigations or during open or laparoscopic surgeries $(5-7,13)$. Abdominal computed tomography (CT), magnetic resonance imaging and scintigraphy with Tc-99 $\mathrm{m}$ are helpful in making the diagnosis of pelvic AS $(14,17)$. Ota et al (14) concluded that $77.8 \%$ (7/9) of feeding vessels of a pelvic AS originated from the great omentum or splendid hilum, and thus, delineating the feeding blood vessels on dynamic CT may be useful for diagnosing AS in the pelvis. For most pelvic AS, regular monitoring by ultrasound inspection is recommended instead of invasive over-treatment. Abdominal sonography and Doppler imaging may be applied to avoid unnecessary examinations if ectopic spleen is considered as a differential diagnosis of pelvic masses (7-9). In addition, tumor markers including CEA and CA199 are always within normal limits in patients with $\mathrm{AS}$, which allows for ruling out any malignant gynecological diseases. Owing to the fact that in the present case, the pelvic masses was suspected to be a malignancy prior to surgery and due to their deep localization, open surgery was preferred. Although open surgery has been the most frequently reported method in previously published reports, the laparoscopic approach may also be used for the diagnosis and treatment of large AS. This approach also enables the ability to observe new information in the abdominal cavity that may not be visible by pre-operative diagnostic imaging alone.

In conclusion, the clinician should be aware of the rare possibility of a pelvic AS in patients who present with adnexa, pelvic discomfort or a pelvic mass. Pelvic AS is finally diagnosed during radiological investigations, or during open or laparoscopic surgeries.

\section{Acknowledgements}

Not applicable.

\section{Funding}

This study was supported by grants from the National Natural Foundation of China (grant no. 81172467) and the Foundation of Health and family Planning Commission of Hubei Province (grant no. WJ2015MB153).

\section{Availability of data and materials}

All data generated or analyzed during this case are included in this published article.

\section{Authors' contributions}

YF and YS contributed to drafting the manuscript. BW and JL contributed to the data collection of the case. SW and DM contributed to the interpretation of the data as well as the editing of the manuscript. MW conceived and designed the study and drafted and revised the manuscript. All authors have critically reviewed this manuscript and approved the last version.

\section{Ethics approval and consent to participate}

The patient provided their written informed consent for inclusion within the present study.

\section{Consent for publication}

The patient provided their written consent for the publication of their data as part of the present study.

\section{Competing interests}

The authors declare that they have no competing interests. 


\section{References}

1. Dodds WJ, Taylor AJ, Erickson SJ, Stewart ET and Lawson TL: Radiologic imaging of splenic anomalies. AJR Am J Roentgenol 155: 805-810, 1990.

2. Zhou JS, Chen X, Zhu T, Ding GJ and Zhang P: Pelvic accessory spleen caused dysmenorrhea. Taiwan J Obstet Gynecol 54: 445-446, 2015.

3. Halpert B and Gyorkey F: Lesions observed in accessory spleens of 311 patients. Am J Clin Pathol 32: 165-168, 1959.

4. Hsiao SM, Lee LC and Chang MH: Large pelvic accessory spleen mimicking an adnexal malignancy in a teenage girl. J Formos Med Assoc 100: 565-567, 2001.

5. Taskin MI, Baser BG, Adali E, Bulbul E and Uzgoren E: Accessory spleen in the pelvis: A case report. Int J Surg Case Rep 12: 23-25, 2015.

6. Perin A, Cola R and Favretti F: Accessory wandering spleen: Report of a case of laparoscopic approach in an asymptomatic patient. Int J Surg Case Rep 5: 887-889, 2014.

7. Vural M, Kacar S, Koşar U and Altin L: Symptomatic wandering accessory spleen in the pelvis: Sonographic findings. J Clin Ultrasound 27: 534-536, 1999.

8. Tendler R, Farah RK, Kais M, Odeh M and Bornstein J: Symptomatic pelvic accessory spleen in a female adolescent: Case report. J Clin Ultrasound 45: 600-602, 2017.

9. Iorio F, Frantellizzi V, Drudi FM, Maghella F and Liberatore M: Locally vascularized pelvic accessory spleen. J Ultrasound 19 : $141-144,2015$

10. Tu C, Lin Q, Zhu J, Shao C, Zhang K, Jiang C, Ding Z, Zhou X, $\mathrm{Tu} \mathrm{J}, \mathrm{Zhu} \mathrm{W}$ and Chen W: Isolated sarcoidosis of accessory spleen in the greater omentum: A case report. Exp Ther Med 11: 2379-2384, 2016.
11. Unver Dogan N, Uysal II, Demirci S, Dogan KH and Kolcu G: Accessory spleens at autopsy. Clin Anat 24: 757-762, 2011.

12. Wood TW and Mangelson N: Urological accessory splenic tissue. J Urol 137: 1219-1220, 1987.

13. Obuchi T, Takagane A, Sato K, Yonezawa H, Funato O and Kobayashi M: Single-incision laparoscopic excision of symptomatic accessory spleen in the pelvis: An initial report. J Minim Access Surg 13: 321-322, 2017.

14. Ota H, Ojima Y, Sumitani D and Okajima M: Dynamic computed tomography findings of an accessory spleen in the pelvis: A case report. Surg Case Rep 2: 23, 2016.

15. Cowles RA and Lazar EL: Symptomatic pelvic accessory spleen. Am J Surg 194: 225-226, 2007.

16. d'Halluin G, Menard J, Dessard P, Dauphin H, Deshayes M, Pierre F and Magnin G: Torsion of the accessory spleen: An atypical etiology for acute abdomen. Gynecol Obstet Fertil 29: 821-823, 2001 (In French).

17. Nishiguchi S, Habu D, Ishizu H, Iwata Y, Tatsumi N, Enomoto M, Minamitani S, Fukuda K, Tamori A, Takeda T, et al: Accessory spleen in the pelvis diagnosed by Tc-99m phytate scintigraphy. Ann Nucl Med 15: 263-265, 2001.

18. Azar GB, Awwad JT and Mufarrij IK: Accessory spleen presenting as adnexal mass. Acta Obstet Gynecol Scand 72: 587-588, 1993.

19. Chen JS, Lin CL, Tsai CC, Lee KT, Ker CG and Sheen PC: Giant ectopic pelvic spleen: Report of a case and review of the literature. Gaoxiong Yi Xue Ke Xue Za Zhi 9: 54-60, 1993. 